\title{
The nonsurgical nature of patients with subarachnoid or intraparenchymal hemorrhage associated with mild traumatic brain injury
}

\author{
Benjamin J. Ditty, MD, ${ }^{1}$ Nidal B. Omar, BS, BA, ${ }^{2}$ Paul M. Foreman, MD, ${ }^{1}$ Daxa M. Patel, MD, \\ Patrick R. Pritchard, MD, ${ }^{1}$ and Mamerhi O. Okor, MD ${ }^{1}$ \\ ${ }^{1}$ Department of Neurosurgery and ${ }^{2}$ School of Medicine, University of Alabama at Birmingham, Alabama
}

\begin{abstract}
OBJECT Mild traumatic brain injury (mTBI), as defined by Glasgow Coma Scale (GCS) score of 13 or higher, is a common problem in the United States and worldwide, estimated to affect more than 1 million patients yearly. When associated with intracranial hemorrhage, it is a common reason for neurosurgical consultation and transfer to tertiary care centers. The authors set out to investigate the clinical implications of subarachnoid hemorrhage (SAH) and/or intraparenchymal hemorrhage (IPH) associated with $\mathrm{mTBI}$ in hopes of standardization of $\mathrm{mTBI}$ clinical care and optimization of resource allocation.
\end{abstract}

METHODS The authors performed a retrospective review of 500 consecutively treated patients with $\mathrm{mTBI}$ and SAH and/or IPH admitted to a Level I trauma center in Alabama between May 2003 and May 2013. They performed a review of medical records to confirm the diagnosis, determine neurological condition at admission, and assess for episodes of neurological decline or brain injury-related complications including altered mental status, seizures, and hyponatremia.

RESULTS Of the 500 patients for whom data were reviewed, 304 (60.8\%) were male and $196(39.2 \%)$ were female. Average age was 46.3 years. Overall, 63 patients (12.6\%) had isolated IPH, $411(82.2 \%)$ had isolated SAH, and $26(5.2 \%)$ had radiographic evidence of both IPH and SAH. One hundred forty-five patients (29\%) were transferred an average distance of 64.5 miles. The authors identified no patients who experienced neurological worsening during their hospital course. Two patients experienced hyponatremia that required treatment with sodium supplementation.

CONCLUSIONS Patients with the constellation of SAH and/or IPH and mTBI do not require neurosurgical consultation, and these findings should not be used as the sole criteria to justify transfer to tertiary referral centers.

http://thejns.org/doi/abs/10.3171/2014.10.JNS132713

KEY WORDS mild traumatic brain injury; subarachnoid hemorrhage; intraparenchymal hemorrhage; contusion; vascular disorders

$\mathrm{M}$ ILD traumatic brain injury (mTBI), defined as a closed-head injury in a patient with a clinical neurological condition consistent with a Glasgow Coma Scale (GCS) score of 13 or greater, is a common condition encountered in medical centers worldwide. The Centers for Disease Control and Prevention (CDC) estimates that mTBI accounts for up to $75 \%$ of the more than 1.5 million cases of TBI reported annually, though the actual incidence may be much greater. ${ }^{10}$ Despite its impressive incidence, intracranial pathology is identified in fewer than $10 \%$ of mTBI patients, and fewer than $1 \%$ receive neurosurgical intervention. ${ }^{6,11}$

Clinical practice guidelines vary based on initial clini- cal assessment, but evaluation of patients with mTBI often includes cranial imaging, generally CT scanning without a contrast agent, to screen for injury. ${ }^{13}$ Imaging may demonstrate no radiographic evidence of mTBI, or it may show mild intracranial injury including small intraparenchymal contusions, subarachnoid hemorrhage (SAH), thin subdural or epidural hematomas, or more substantial intracranial hemorrhage. In the absence of additional injuries requiring hospitalization, patients with a negative head CT scan are likely to be discharged to home without additional observation or repeat brain imaging; patients with mild intracranial injury are likely to be observed overnight, even if that requires transfer to a higher level of care; and patients with

ABBREVIATIONS CDC = Centers for Disease Control and Prevention; GCS = Glasgow Coma Scale; ICD-9 = International Classification of Diseases, Ninth Revision; INR = International Normalized Ratio; IPH = intraparenchymal hemorrhage; $\mathrm{mTBI}$ = mild traumatic brain injury; $\mathrm{SAH}$ = subarachnoid hemorrhage.

SUBMITTED January 16, 2014. ACCEPTED October 27, 2014.

INCLUDE WHEN CITING Published online December 19, 2014; DOI: 10.3171/2014.10.JNS132713.

DISCLOSURE The authors report no conflict of interest concerning the materials or methods used in this study or the findings specified in this paper. 
more substantial imaging findings may require intense monitoring in the short term.

It is most likely that patients with relatively modest cranial imaging findings (SAH or small intraparenchymal contusions), particularly those not taking antithrombotic medications, are unlikely to experience deterioration and require medical or surgical intervention. A number of recent studies in the trauma and neurosurgical literature have echoed this observation, ${ }^{1-3,12,15}$ which is significant because it brings to light the issue of appropriate and responsible utilization of health care resources in the context of a common medical condition. There are limited data on direct and indirect cost of mTBI, but in its 2003 annual report to the United States Congress, the CDC noted that mTBI costs the United States at least \$17 billion per year. ${ }^{10}$ Given that therapeutic and morbidity-related costs are not expected to be as high in mTBI as in moderate (GCS score of 9-12) to severe (GCS score of $\leq 8$ ) TBI, it is probable that a significant share of the economic impact is related to hospital admissions and workup that may not always be warranted. For example, repeat intracranial imaging is commonly performed in patients with mild intracranial hemorrhage. However, a recent meta-analysis demonstrated that repeat imaging rarely reveals lesions requiring a change in clinical management, and there is a lack of statistical evidence supporting repeat imaging in patients who are clinically stable or improved. ${ }^{2}$ Indiscriminate reimaging in the setting of mTBI creates both a cost/benefit and risk/benefit dilemma.

Another issue related to allocation of resources is the overuse of neurosurgical consultation. At a busy center with a large volume of trauma cases, neurosurgical services can be overwhelmed with consult requests for patients who are highly unlikely to require medical or surgical intervention for their brain injuries. These consult requests can occupy the limited time of on-call neurosurgeons and distract their attention from patients with more acute neurological illnesses. A recent cross-sectional study of trauma patients at an academic Level I trauma center demonstrated that of $75 \mathrm{mTBI}$ patients admitted with isolated $\mathrm{SAH}$, only one experienced clinical deterioration and this was due to pulmonary rather than neurological complications. ${ }^{3}$ The study's authors suggested that the lack of guidelines informing logistical decisions in the management of these patients has resulted in neurosurgical consultation becoming the standard of care. ${ }^{3}$ A related practice that often consumes an even greater share of time and resources is the transfer of patients suffering mTBI from hospitals that lack neurosurgical services. The resulting health care provision difficulty is that the transfer to Level I trauma centers may overwhelm the hospital capacity and result in the hospital's being placed on diversion. This eliminates the ability to accept and care for patients who truly are at risk for neurological deterioration. These transfers are certainly warranted when a true surgical lesion has been identified, but many transfers are spurred by the uneasiness of those in the emergency department or by other physicians concerned about a patient's potential decline, despite literature demonstrating a very low incidence of delayed deterioration in patients with mTBI. ${ }^{4}$ With the growing body of data pointing out the low likelihood that neurosurgical intervention will be necessary in mTBI, there is a need for the development of a clinical pathway to triage these patients for more efficient treatment.

It is the goal of this study to investigate the clinical implications of mTBI in an attempt to distinguish those patients who are at risk for neurological decline from those who are not. This information will be interpreted in the context of medical resource allocation to provide physicians an evidence-based paradigm for the management of these patients. This paradigm should be applicable by any practitioner with a general medical knowledge and not require a neurosurgical background to interpret.

\section{Methods}

The trauma registry at a Level I trauma center was reviewed to identify a convenience sample of 500 consecutively treated patients with a mTBI (GCS score $\geq 13$ ) and International Classification of Diseases, Ninth Revision (ICD-9) diagnoses of SAH and/or intraparenchymal contusions (ICD-9 codes 852.0 and 851.0, respectively) and excluded patients with diagnoses of subdural or epidural hematoma (ICD-9 codes 852.2-852.3 and 852.4-852.5, respectively). The accuracy of the diagnoses was determined based on concordance between the radiology report and neurosurgical consult note; if there was disagreement, images were reviewed and if a definitive conclusion could not be reached, the patient was excluded from the study. Patients with a penetrating cranial injury, depressed skull fracture, CSF leak, evidence of aneurysmal SAH, delayed presentation, or fatal extracranial injuries were also excluded.

Medical records were evaluated to determine each patient's hospital course, particularly their neurological status on admission and whether any episodes of neurological decline including altered mental status, focal neurological deficit, or seizure occurred during the hospitalization, particularly during the 1st hospital day, after which, in the absence of neurological deterioration, neurosurgical services typically cease following these patients. Special attention was paid to episodes of altered mental status or episodes consistent with epileptic activity. Medical records were also reviewed to identify whether patients had been taking anticoagulant or antiplatelet medications prior to their injury.

Transfer distances were determined using Google Maps and the published street addresses on the transferring hospitals' public websites. Information regarding general transfer statistics and costs was obtained from the office of our institution's Critical Care Transport service.

\section{Results}

We identified 500 patients with mTBI admitted to the University of Alabama Hospital between May 2003 and May 2013. Of these 500 patients, 304 (60.8\%) were male and $196(39.2 \%)$ were female (Table 1). The mean age $( \pm \mathrm{SD})$ of these patients was $46.3 \pm 18.8$ years (range 18.1-93.6 years) (Table 1). The GCS score at presentation was 15 in 462 patients $(92.4 \%), 14$ in $30(6 \%)$, and 13 in 8 (1.6\%) (Table 2). CT scanning revealed evidence of isolated SAH in 411 patients $(82.2 \%)$, isolated intraparenchymal 
TABLE 1. Demographic data obtained in 500 patients with mTBI associated with SAH and/or IPH admitted between May 2003 and May 2013

\begin{tabular}{cc}
\hline \multicolumn{1}{c}{ Factor } & Value (\%) \\
\hline Sex & \\
\hline Female & $196(39.2)$ \\
\hline Male & $304(60.8)$ \\
\hline Age & 46.3 \\
\hline Mean & 18.8 \\
\hline Standard deviation & $18.1-93.6$ \\
\hline Range & $411(82.2)$ \\
\hline Hemorrhage type & $63(12.6)$ \\
\hline SAH & $26(5.2)$ \\
\hline IPH &
\end{tabular}

hemorrhage (IPH) in 63 (12.6\%), and both SAH and IPH in $26(5.2 \%)$ (Table 1).

Of the 469 patients for whom prehospital medication lists were available, 71 patients were prescribed at least one antiplatelet or anticoagulant medication. Forty-five patients reported taking only aspirin, 22 patients a combination of aspirin and clopidogrel (Plavix), and 3 patients a single antiplatelet agent other than aspirin. Only 1 patient was taking Coumadin; no other patients reported anticoagulant use (Table 3).

One hundred fifty-six patients were transferred from referring hospitals. For the 145 patients for whom data on the referring hospitals were available, mean transfer distance was 64.5 miles. The shortest transfer distance was 0.3 miles and the greatest transfer distance was 262 miles. Although data were not available for mode of transport of individual patients, the Critical Care Transport office at our institution estimates an overall air to ground transport ratio of 1:3. Gross charges for ground transport are $\$ 1200$ plus an additional $\$ 22$ per mile, while gross charges for air transport are $\$ 7500$ plus an additional $\$ 54$ per mile. Other charges apply based on the services or treatments patients receive en route.

The mean $( \pm$ SD) hospital stay was $3.3 \pm 3.7$ days (range 1-46 days); $92 \%$ of patients were admitted for $\leq 7$ days, and the 5 patients with the longest stays were all over the age of 65 years and had extensive orthopedic, intrathoracic, or intraabdominal pathology. No patients experienced neurological decline during their hospitalization, nor did any of these patients experience seizures during their hospital stay. Two patients were found to have hyponatremia at some point during their hospitalization. Both had a GCS score of 15 on arrival. One patient's initial serum sodium

TABLE 2. Summary of GCS scores stratified by time

\begin{tabular}{ccc}
\hline & \multicolumn{2}{c}{ No. of Patients (\%) } \\
\cline { 2 - 3 } GCS Score & Emergency Department & Hospital Day 1 \\
\hline 13 & $8(1.6)$ & $0(0)$ \\
\hline 14 & $30(6)$ & $0(0)$ \\
\hline 15 & $462(92.4)$ & $500(100)$ \\
\hline
\end{tabular}

TABLE 3. Anticoagulant status

\begin{tabular}{lc}
\hline \multicolumn{1}{c}{ Status } & No. of Patients \\
\hline No preinjury anticoagulant use & 398 \\
\hline Unknown blood thinner status & 31 \\
\hline Presence of preinjury anticoagulant use & \\
\hline Aspirin & 45 \\
\hline Aspirin + clopidogrel & 22 \\
\hline Clopidogrel & 1 \\
\hline Coumadin & 1 \\
\hline Aggrenox & 1 \\
\hline Ticlopidine & 1 \\
\hline
\end{tabular}

level was $121 \mathrm{mEq} / \mathrm{L}$, presumably due to chronic hyponatremia. This patient's serum sodium was slowly corrected to a normal level. The other patient had a one-time serum sodium level of $129 \mathrm{mEq} / \mathrm{L}$ mid-hospitalization and was started on sodium supplementation, which normalized the laboratory value. No patient returned requiring delayed neurosurgical intervention following discharge.

\section{Discussion}

The results of this study support the position that neurosurgical intervention is highly unlikely in mTBI patients who sustain SAH or IPH. The two patients who developed hyponatremia were treated with hypertonic saline, which could easily have been administered by any medical service and did not require neurosurgical expertise. Approximately $31 \%$ of the patients in our study were transferred from outside hospitals. For the 145 patients for whom transfer distance was readily available, we calculated an average distance of 64.5 miles per transfer, with the greatest distance being 262 miles. Based on the gross charges presented earlier, this translates into an average of a \$2619-\$10,983 minimum cost per transfer depending on the mode of transport and costs upwards of $\$ 21,648$ for the greatest transfer distances. In addition to the cost of transport, every neurosurgical consult generates a charge for the provision of initial inpatient care, which is reimbursed at rates of \$92.72-\$185.89 for patients with Medicare, \$88$\$ 202$ for those with Medicaid, and \$82.88-\$155.68 and \$182.28-\$363.83 for those with Blue Cross/Blue Shield and United Healthcare insurance policies, respectively. The economic impact of these costs on the health care system cannot be discounted. Considering these costs, as well as the large volume of acutely ill patients on neurosurgical services at tertiary care medical centers, it is our hope that the findings of this study serve to reiterate the fact that a certain subset of patients presenting with mTBI can safely be managed without a neurosurgical consultation or transfer from secondary care hospitals. There is a need for standardization in the triage of mTBI patients that takes into account the growing body of outcomes data so that more effective allocation of resources can be achieved.

Clinical practice guidelines set forth by the CDC and American College of Emergency Physicians discuss in depth the indications for imaging in mTBI and discharging patients with negative CT scans but do not provide a unified approach to patients in whom there is evidence of 
mild intracranial bleeds. ${ }^{7}$ Despite the absence of evidencebased guidelines for the logistics of managing mTBI patients with small intracranial bleeds, recommendations have been published in the literature. Carlson et al. have proposed an algorithm for the triage of mTBI patients at hospitals without neurosurgical services based on teleradiology. Indications for transfer included evidence of operative intracranial pathology according to the accepting neurosurgeon or no operative lesion but the presence of an epidural hematoma, contusion $>2 \mathrm{~cm}$ in greatest diameter, age over 70 years, clopidogrel use prior to injury, or International Normalized Ratio (INR) > 1.5. ${ }^{4}$ A University of Michigan study by Wu et al. reiterated the low incidence of neurosurgical intervention in mTBI patients and suggested using factors such as suspected CSF leak, coagulopathy, depressed skull fracture, blood in the basal cisterns or sylvian fissure, or evidence of progressive hemorrhage on repeat CT scan, to determine the need for neurosurgical consultation. ${ }^{15}$ In a study analyzing neurosurgical consultation at UC Davis Medical Center, van Essen et al. ${ }^{14}$ considered neurosurgical consultation for adult head injury appropriate if one or more high-risk criteria of the Canadian CT Head Rule were met, or if the patient fell into the high-risk group for intracranial injury defined by Masters et al., i.e., depressed level of consciousness not attributable to other endogenous or exogenous causes, focal neurological signs, decreasing level of consciousness, penetrating skull wound, or palpable depressed fracture..$^{14}$ Borczuk et al. proposed that patients with a GCS score of 15 and isolated SAH on CT may not warrant transfer from hospitals without neurosurgical services because there is a low risk of deterioration. ${ }^{3}$

Based on the results of the present study, considered in the context of prior research on this topic, it is reasonable to assume that neurosurgical consultation is unnecessary in patients presenting with a GCS score of $\geq 13$ and CT evidence of isolated SAH and/or IPH. These patients may require admission for management of other injuries, but if the radiological intracranial finding represents the sole lesion identified, the data suggest that the patients can safely be discharged after evaluation by an emergency department physician or other initial treating physician. Additionally, patients suffering mTBI with SAH and/or IPH who present to secondary care hospitals do not warrant transfer to tertiary care centers if their other injuries can be managed at the secondary care hospitals.

Whether a patient's antiplatelet or anticoagulant intake should influence triage for neurosurgical consultation remains a valid question. Seventy-one of the 500 patients included in this study were known to be taking antiplatelet or anticoagulant agents prior to injury, with the majority taking aspirin and a nontrivial number taking a combination of aspirin and clopidogrel (Table 1). Because none of the patients in our study experienced any adverse neurological events, we found no correlation between a patient's antiplatelet or anticoagulant status and risk for neurological decline. The aforementioned University of Michigan study demonstrated a lack of statistical significance in the correlation between aspirin or clopidogrel use and the need for neurosurgical intervention in mTBI patients with mild intracranial findings. ${ }^{15}$ The previously mentioned study by
Carlson et al. ${ }^{4}$ suggests that clopidogrel use or an INR > 1.5 should be included in the criteria for transfer of mTBI patients from facilities lacking neurosurgical services but does not elaborate on the rationale for this recommendation. A study conducted by the Italian Society of Emergency Medicine (also known as SIMEU) analyzed data from 1558 patients with different degrees of intracranial injury and found a cumulatively increased risk of worsening lesion on repeat head CT scan, need for neurosurgical intervention, and unfavorable outcome at 6 months for patients on blood thinners, particularly clopidogrel..$^{5}$ With regards to mTBI (defined in their study as GCS score of 14-15), although the incidence of neurological decline was low, for patients taking anticoagulant medication there was a statistically significant increase in neurological decline that became more pronounced with a greater number of intracranial hemorrhages..$^{5}$ The data mining analysis in this study did not specifically look at outcomes related to both mTBI and the specific subtypes of intracranial hemorrhage; however, it should be noted that for all severities combined with the subtypes of EDH, traumatic SAH, and IVH did not independently predict worsening with statistical significance. ${ }^{5}$ Further research is needed to determine whether specific anticoagulants or an INR cutoff should be factored into the triage of mTBI patients.

With the mounting degree of evidence to support decreased utilization of neurosurgical consultation and transfers for patients with mTBI and mild intracranial findings, it is important for safeguards to be in place for the rare patient who may experience deterioration and subsequently require neurosurgical intervention. In hospitals that lack round-the-clock neurosurgical services, there have been a number of studies supporting the increased use of teleradiology.,9 A neurosurgeon's review, at an accepting facility, of transmitted neuroimages is not only helpful for initially determining if a patient needs to be transferred but also for reevaluating if this is necessary. There has also been increased discussion in the trauma literature about the acute care surgeons and their role in the management of TBI. In a retrospective analysis of 270 mTBI patients with positive CT findings at the University of Arizona, the authors found that of the 90 patients who received neurosurgical consultation and 180 patients who received management by acute care surgery personnel without consultation, no patient required neurosurgical intervention or suffered in-hospital mortality. ${ }^{8}$ The study also demonstrated a statistically significant reduction in hospital costs by avoiding unnecessary neurosurgical consultation (mean hospital costs of $\$ 14,108$ vs $\$ 9400$ per patient for those who received a neurosurgical consult vs those who did not, respectively). ${ }^{8}$ Based on an analysis of TBI data over the past 5 years, the acute care surgical team at the University of Arizona now independently manages isolated SAH and contusions as well as epidural/subdural hematomas of less than or equal to $4 \mathrm{~mm}^{8}{ }^{8}$

Limitations of the present study include its retrospective design and lack of a control group. That being said, this study represents a vital step in facilitating future prospective research in this area. Given the current widespread practice of neurosurgical consultation or transfer to centers with neurosurgical expertise for the patient 
population described in this study, it is our expectation that outcomes data from the present study and numerous others demonstrating the lack of neurological decline or requirement for neurosurgical intervention will encourage emergency physicians and trauma surgeons to partake in prospective studies on the management of these patients.

Another limitation of this study is the greater proportion of patients with a GCS score of 15 versus those with a GCS score of 13 or 14 . On presentation to the emergency department, $92.4 \%$ of patients had a GCS score of $15,6 \%$ had a GCS score of 14, and the remaining $1.6 \%$ had a GCS score of 13 (Table 2). This does not reflect a sampling bias; because we used data from consecutive patients, it demonstrates the fact that a majority of mTBI patients with SAH and/or IPH will present with a GCS score of 15 . There is, however, an overall greater amount of data in the literature backing benign outcomes in mTBI patients with a GCS score of 15. Although our research suggests that neurosurgical intervention is unlikely in mTBI regardless of the GCS score, further research involving patients with a GCS score of 13 or 14 would be needed to elucidate whether the specific GCS score plays any role in outcomes. However, it is clear that withholding neurosurgical consultation for mTBI patients with a GCS score of 15 alone would significantly reduce the burden of unnecessary consultations while not having an impact on quality of care for patients and also aiding in optimal allocation of health care resources.

Despite the fact that no patient in the present study required delayed neurosurgical intervention, these recommendations do not address the potential need for some patients with head injuries, in particular the elderly, to undergo repeat CT imaging in a delayed fashion (2-4 weeks) to evaluate for the development of a delayed subdural hematoma.

\section{Conclusions}

This is currently the largest retrospective study on short-term outcomes in mTBI patients who have suffered an SAH and/or IPH, and it demonstrates the nonsurgical nature of this condition, strengthening the growing body of literature advocating the need for triage guidelines that yield a more responsible and efficient allocation of resources. These data suggest that trauma patients with a GCS score of 13-15 on initial evaluation and imaging evidence of traumatic SAH and/or an intraparenchymal contusion do not require neurosurgical consultation or transfer to tertiary care centers and can safely be discharged, barring the presence of other injuries or medical issues that require inpatient management.

\section{Acknowledgment}

Dr. Ditty completed this work as a Women's Leadership Council Clinical Scholar in the Department of Neurosurgery at the University of Alabama at Birmingham.

\section{References}

1. AbdelFattah KR, Eastman AL, Aldy KN, Wolf SE, Minei JP, Scott WW, et al: A prospective evaluation of the use of routine repeat cranial CT scans in patients with intracranial hemor- rhage and GCS score of 13 to 15 . J Trauma Acute Care Surg 73:685-688, 2012

2. Almenawer SA, Bogza I, Yarascavitch B, Sne N, Farrokhyar F, Murty N, et al: The value of scheduled repeat cranial computed tomography after mild head injury: single-center series and meta-analysis. Neurosurgery 72:56-64, 2013

3. Borczuk P, Penn J, Peak D, Chang Y: Patients with traumatic subarachnoid hemorrhage are at low risk for deterioration or neurosurgical intervention. J Trauma Acute Care Surg 74:1504-1509, 2013

4. Carlson AP, Ramirez P, Kennedy G, McLean AR, MurrayKrezan C, Stippler M: Low rate of delayed deterioration requiring surgical treatment in patients transferred to a tertiary care center for mild traumatic brain injury. Neurosurg Focus 29(5):E3, 2010

5. Fabbri A, Servadei F, Marchesini G, Bronzoni C, Montesi D, Arietta L: Antiplatelet therapy and the outcome of subjects with intracranial injury: the Italian SIMEU study. Crit Care 17:R53, 2013

6. Haydel M: Management of mild traumatic brain injury in the emergency department. Emerg Med Pract 14:1-24, 2012

7. Jagoda AS, Bazarian JJ, Bruns JJ Jr, Cantrill SV, Gean AD, Howard PK, et al: Clinical policy: neuroimaging and decisionmaking in adult mild traumatic brain injury in the acute setting. Ann Emerg Med 52:714-748, 2008

8. Joseph B, Aziz H, Sadoun M, Kulvatunyou N, Tang A, O'Keeffe T, et al: The acute care surgery model: managing traumatic brain injury without an inpatient neurosurgical consultation. J Trauma Acute Care Surg 75:102-105, 2013

9. Klein Y, Donchik V, Jaffe D, Simon D, Kessel B, Levy L, et al: Management of patients with traumatic intracranial injury in hospitals without neurosurgical service. J Trauma 69:544-548, 2010

10. National Center for Injury Prevention and Control: Report to Congress on Mild Traumatic Brain Injury in the United States: Steps to Prevent a Serious Public Health Problem. Atlanta: Centers for Disease Control and Prevention, 2003 (http://www.cdc.gov/ncipc/pub-res/mtbi/mtbireport.pdf) [Accessed October 28, 2014]

11. Pandor A, Goodacre S, Harnan S, Holmes M, Pickering A, Fitzgerald P, et al: Diagnostic management strategies for adults and children with minor head injury: a systematic review and an economic evaluation. Health Technol Assess 15:1-202, 2011

12. Quigley MR, Chew BG, Swartz CE, Wilberger JE: The clinical significance of isolated traumatic subarachnoid hemorrhage. J Trauma Acute Care Surg 74:581-584, 2013

13. Tavender EJ, Bosch M, Green S, O'Connor D, Pitt V, Phillips K, et al: Quality and consistency of guidelines for the management of mild traumatic brain injury in the emergency department. Acad Emerg Med 18:880-889, 2011

14. van Essen TA, Heeringa JJ, Muizelaar JP: (In)appropriate neurosurgical consultation. Clin Neurol Neurosurg 112:775-780, 2010

15. Wu C, Orringer DA, Lau D, Fletcher JJ: Cumulative incidence and predictors of neurosurgical interventions following nonsevere traumatic brain injury with mildly abnormal head imaging findings. J Trauma Acute Care Surg 73:1247-1253, 2012

\section{Author Contributions}

Conception and design: Okor, Ditty, Pritchard. Acquisition of data: Ditty, Omar. Analysis and interpretation of data: Ditty, Omar. Drafting the article: Ditty, Omar. Critically revising the article: all authors. Reviewed submitted version of manuscript: all authors. Study supervision: Okor, Ditty, Pritchard.

\section{Correspondence}

Mamerhi O. Okor, Department of Neurosurgery, 510 20th St. S., FOT 1034, Birmingham, AL 35294.email: mookor@uabmc.edu. 